Scheme I

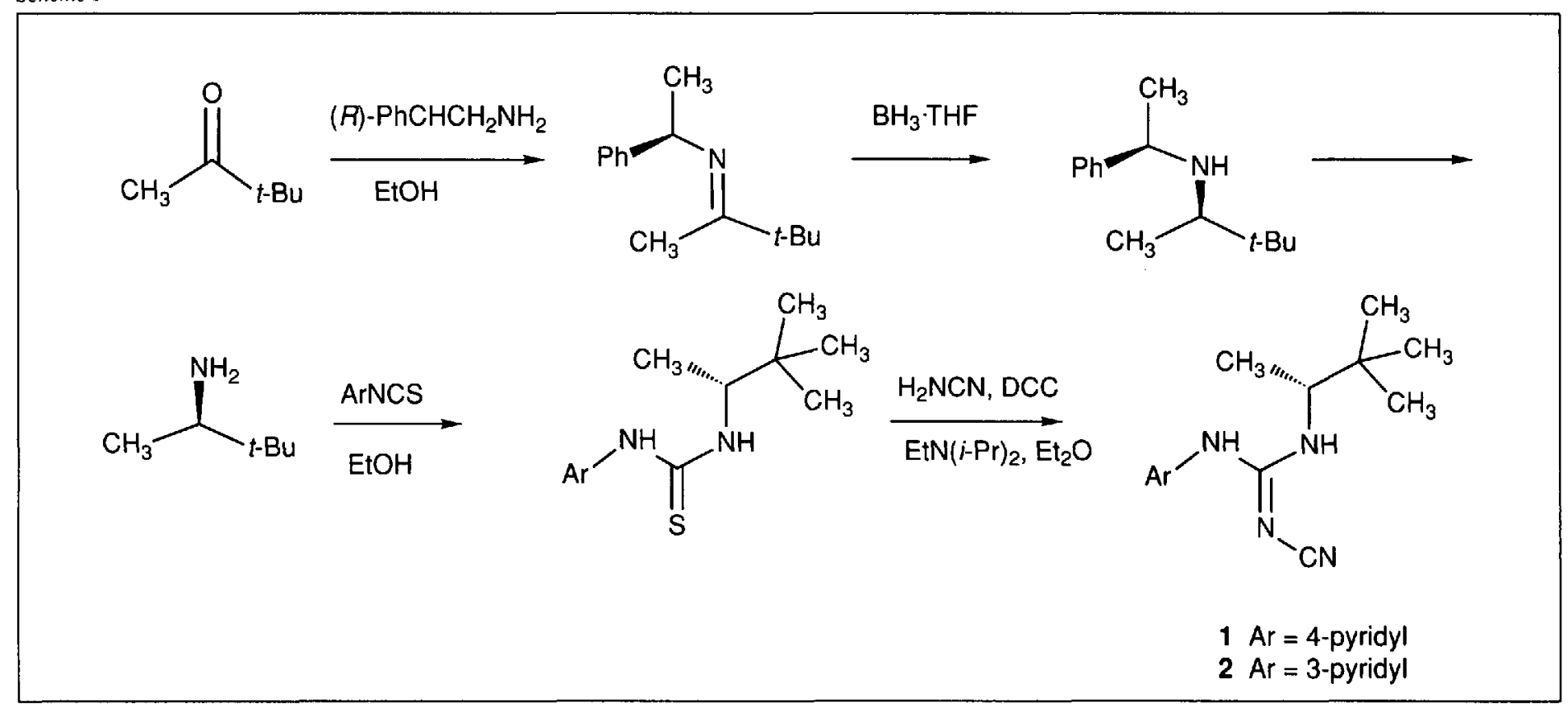

Scheme 2

bition of spontaneous mechanical activity $\left(\mathrm{p} / C_{50}\right)$ and stimulation of ${ }^{86} \mathrm{Rb}-\mathrm{efflux}\left(\mathrm{p} E C_{15}\right)$ in rat portal veins [6], and revealed that $\mathrm{K}$ channel opening activity was stereoselective with $(R)$-pinacidil $\left(\mathrm{p} / C_{50}=7.6\right.$ ) being 12 times more potent than $(S)$-pinacidil $\left(\mathrm{p} / C_{50}=\right.$ 6.1). Similar stereoselectivity was found for the 3-pyridyl analogues of pinacidil (2). Paradoxically, however, with the nitro- ethenediamines, as illustrated for the 3-pyridyl analogue 4, the stereoselectivity for $\mathrm{K}$ channel opening was reversed, with the $(S)$ enantiomer $\left(\mathrm{p} / C_{50}=8.0\right)$ being 100 -fold more active than its corresponding $(R)$-enantio$\operatorname{mer}\left(\mathrm{p} / C_{50}=6.0\right)$.

(Abstract by the authors)

Chimia 45 (1991) 89-90

(C) Schweiz. Chemiker-Verband; ISSN $0009-4293$

\section{The Search for Peptidoleukotriene Antagonists}

Andreas von Sprecher*, Alfred Sallmann, Andreas Beck, Werner Breitenstein, Hansruedi Wiestner, Sabine Kimmel, Wayne H. Anderson, Gary P. Anderson, Natarajan Subramanian, and Michael A. Bray

The peptidoleukotrienes $\mathrm{LTC}_{4}, \mathrm{LTD}_{4}$, and $\mathrm{LTE}_{4}$ are thought to play a major role in allergic asthma, due to their potent bronchoconstrictor and inflammatory properties. The first leukotriene (LT) antagonist,

FPL55712, was discovered in 1973 six years before the structures of the LT's were defined by Samuelsson and Corey. Initial chemical approaches to the discovery of new LT antagonists were based mainly on the
Received: November 30, 1990

[1] H.J. Petersen, C.K. Nielsen, E. Arigoni-Martelli, J. Med. Chem. 1978, 21, 773.

[2] N.S. Cook, U. Quast, P.W. Manley, Br. $J$. Pharmacol. 1989, 96, $181 \mathrm{P}$.

[3] R. Ganellin, J. Med. Chem. 1981, 24, 913.

[4] E. Arigoni-Martelli, C.K. Nielsen, U.B. Olsen, H.J. Petersen, Experientia 1980, 36, 445.

[5] R.Gompper, H.Schaefer, Chem. Ber. 1967,100, 591.

[6] U. Quast, Br. J. Pharmacol. 1987, 9I, 569.

structure of FPL55712 and, after 1980, on the structure of $\mathrm{LTD}_{4} . L Y 171883, L-648051$, Ro23-3544, CGP35949D, and $Y M-16638$ are examples of FPL55712 analogs that are or were in clinical development. However, the clinical data reported so far are not encouraging. These compounds, as well as the first LT analogs, can be considered to be 'first generation' antagonists showing antagonist potency in the range of FPL55712. Recently 'second generation' antagonists with greatly enhanced potency have been

*Correspondence: Dr. A. von Sprecher Research Department, Pharmaceuticals Division Ciba-Geigy Ltd.

CH-4002 Basel 
Scheme I

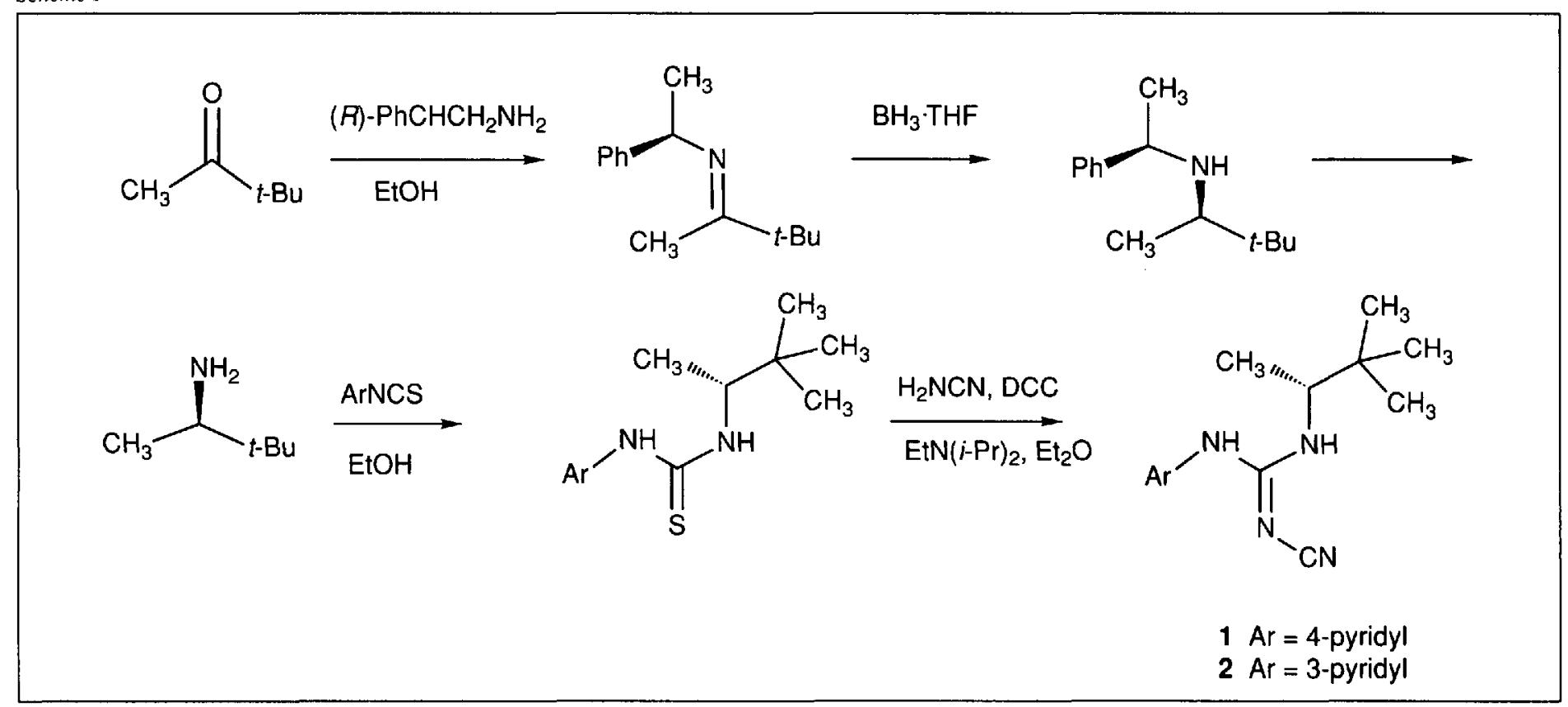

Scheme 2

bition of spontaneous mechanical activity $\left(\mathrm{p} / C_{50}\right)$ and stimulation of ${ }^{86} \mathrm{Rb}-\mathrm{efflux}\left(\mathrm{p} E C_{15}\right)$ in rat portal veins [6], and revealed that $\mathrm{K}$ channel opening activity was stereoselective with $(R)$-pinacidil $\left(\mathrm{p} / C_{50}=7.6\right.$ ) being 12 times more potent than $(S)$-pinacidil $\left(\mathrm{p} / C_{50}=\right.$ 6.1). Similar stereoselectivity was found for the 3-pyridyl analogues of pinacidil (2). Paradoxically, however, with the nitro- ethenediamines, as illustrated for the 3-pyridyl analogue 4, the stereoselectivity for $\mathrm{K}$ channel opening was reversed, with the $(S)$ enantiomer $\left(\mathrm{p} / C_{50}=8.0\right)$ being 100 -fold more active than its corresponding $(R)$-enantio$\operatorname{mer}\left(\mathrm{p} / C_{50}=6.0\right)$.

(Abstract by the authors)

Chimia 45 (1991) 89-90

(C) Schweiz. Chemiker-Verband; ISSN $0009-4293$

\section{The Search for Peptidoleukotriene Antagonists}

Andreas von Sprecher*, Alfred Sallmann, Andreas Beck, Werner Breitenstein, Hansruedi Wiestner, Sabine Kimmel, Wayne H. Anderson, Gary P. Anderson, Natarajan Subramanian, and Michael A. Bray

The peptidoleukotrienes $\mathrm{LTC}_{4}, \mathrm{LTD}_{4}$, and $\mathrm{LTE}_{4}$ are thought to play a major role in allergic asthma, due to their potent bronchoconstrictor and inflammatory properties. The first leukotriene (LT) antagonist,

FPL55712, was discovered in 1973 six years before the structures of the LT's were defined by Samuelsson and Corey. Initial chemical approaches to the discovery of new LT antagonists were based mainly on the
Received: November 30, 1990

[1] H.J. Petersen, C.K. Nielsen, E. Arigoni-Martelli, J. Med. Chem. 1978, 21, 773.

[2] N.S. Cook, U. Quast, P.W. Manley, Br. $J$. Pharmacol. 1989, 96, $181 \mathrm{P}$.

[3] R. Ganellin, J. Med. Chem. 1981, 24, 913.

[4] E. Arigoni-Martelli, C.K. Nielsen, U.B. Olsen, H.J. Petersen, Experientia 1980, 36, 445.

[5] R.Gompper, H.Schaefer, Chem. Ber. 1967,100, 591.

[6] U. Quast, Br. J. Pharmacol. 1987, 9I, 569.

structure of FPL55712 and, after 1980, on the structure of $\mathrm{LTD}_{4} . L Y 171883, L-648051$, Ro23-3544, CGP35949D, and $Y M-16638$ are examples of FPL55712 analogs that are or were in clinical development. However, the clinical data reported so far are not encouraging. These compounds, as well as the first LT analogs, can be considered to be 'first generation' antagonists showing antagonist potency in the range of FPL55712. Recently 'second generation' antagonists with greatly enhanced potency have been

*Correspondence: Dr. A. von Sprecher Research Department, Pharmaceuticals Division Ciba-Geigy Ltd.

CH-4002 Basel 
described. These LT antagonists belong to different structural classes: I) Indazoles and Indoles: ICII98615 and ICI204219. 2) Quinolines: $M K-571, W y 48252, R G 12525$, and $S R 2640.3)$ Miscellaneous structures: ONO-1078. 4) LT analogs: SKF104353, LY170680, and CGP457I5A. CGP457I5A is the most potent and longest acting antagonist of the LT analog type known to us. In vitro, CGP45715A is a potent antagonist of $\mathrm{LTD}_{4}$ induced smooth muscle contractions (guinea pig ileum and lung parenchyma) and shows similar affinity for the guinea pig lung $\mathrm{LTD}_{4}$ receptor as the known industry leaders (ICI2042I9, MK-571, and SKF I04353). In vivo, CGP45715A is an extremely potent and long acting antagonist of $\mathrm{LTD}_{4}$ mediated bronchoconstriction in guinea pigs, when given by aerosol, intravenous, or oral routes of administration. Applied as an aerosol $1 \mathrm{~h}$ prior to $\mathrm{LTD}_{4}$ (aerosol) challenge the $E D_{50}$ is $0.000045 \%$ (concentration of aerosol solution) corresponding to $\sim 30 \mathrm{ng} / \mathrm{kg}\left(S K F 104353 E D_{50}>\right.$ $0.01 \%$; ICI204219ED $E D_{50}=0.0013 \%$ ). CGP45715A is also a potent inhibitor of antigen induced, LT-dependent bronchoconstriction in sensitized guinea pigs. In this model, as well as in the $\mathrm{LTD}_{4}$ challenge model, the duration of action of p.o. administered CGP45715A is at least $24 \mathrm{~h}$.
CGP45715A is the result of extensive structure activity studies in the course of which around $600 \mathrm{LT}$ analogs have been synthesized. CGP45715A is a structural analog of $\mathrm{LTD}_{4}$. The carboxylic group in the eicosanoid chain of $\mathrm{LTD}_{4}$ is replaced by a $\mathrm{CF}_{3}$ group and the dipeptide sulfur side chain by a chromonecarboxylic acid. The eicosanoid backbone could be stabilized by the integration of two $\mathrm{Ph}$ rings: one in the polar region and the other at the lipophilic end of the molecule. The $(1 R, 2 S)$-configuration of CGP457I5A does not correspond to the $(5 S, 6 R)$-configuration of the natural LT's. The $(1 S, 2 R)$-enantiomer displaying the absolute configuration of $\mathrm{LTD}_{4}$ shows only weak $\mathrm{LTD}_{4}$ antagonist activity. Besides the configuration, other structural parameters, such as the length of the backbone, the substitution pattern of the $\mathrm{Ph}$ nuclei, the number of double bonds, and the nature of the sulfur side chain, are critical for optimal $\mathrm{LTD}_{4}$ antagonism.

The clinical evaluation of 'second generation' antagonists will clarify, whether LT antagonists are to be of value in the treatment of allergic asthma and other diseases with possible LT involvement.

(Abstract by the authors)

Received: December II, 1990

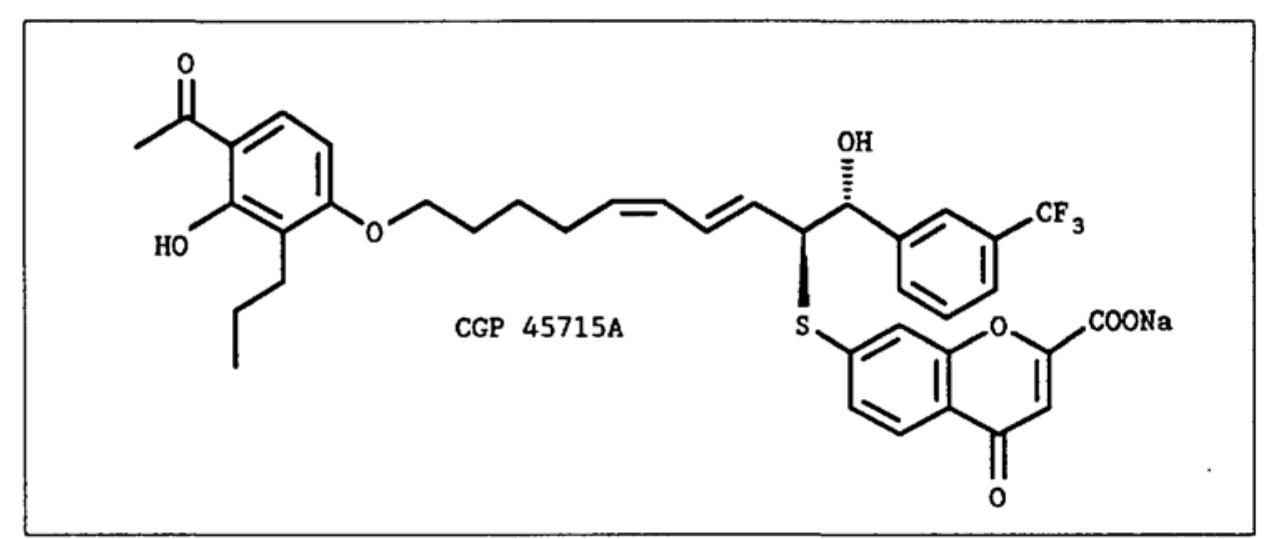

\section{COMPUTATIONAL CHEMISTRY COLUMN}

Column Editors:

Prof. Dr. J. Weber, University of Geneva PD Dr. H. Huber, University of Basel Dr. H. P. Weber, Sandoz AG, Basel

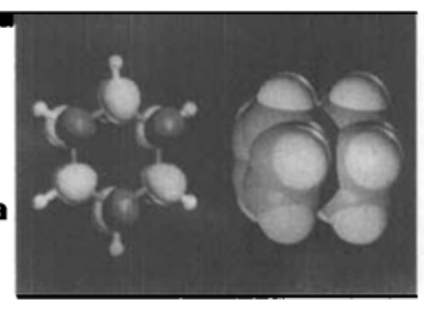

Chimia 45 (1991) 90-92

(c) Schweiz. Chemiker-Verband; ISSN 0009-4293

\section{When Schrödinger and Newton work together...}

\author{
'Man can believe the impossible, but \\ man can never believe the improbable'
}

(Oscar Wilde)

As the invited speaker $H$. Berendsen recently stated in Geneva, the future of molecular simulations lies undoubtedly in mixing more and more Schrödinger with Newton. In other words, realistic simulations of many-particle systems are going to be more and more performed by using Newtonian mechanics with interaction potentials parametrized in part by solving the Schrödinger equation. For quantum chemists proud of Dirac heritage ('the underlying physical laws...'), the pill could seem in a first sight somewhat bitter: why should they struggle hard to devise intricate methodological and computational strategies to solve $\mathrm{H} \Psi=\mathrm{E} \Psi$, if the outcome of their efforts is merely used as an ingredient of the old-fashioned, classical Newtonian mechanics? It is something like asking F1 pilots to run races in order to help car manufacturers to design $2 \mathrm{CV}$ engines..

The impatient reader should not be confused here with our intention: we are in no case suggesting that quantum mechanics is of no practical use in modeling and understanding the behavior of chemical systems. On the contrary, quantum chemistry is probably one of the most successful theories among the whole range of molecular sciences. Clearly, there are numerous examples in the literature where quantum chemistry, often through its simplest models, has brought a decisive contribution to the rationalization of important chemical phenomena, such as the pericyclic reactions (remember the Woodward-Hoffmann rules) or the spectroscopic properties of transitionmetal complexes (remember the modern ligand field theory). However, quantum mechanical techniques are notoriously inadequate for modeling macromolecules or solvated systems, but the problem is here to select a theoretical approach appropriate to the case under study.

Undoubtedly, due to their scaling varying from $N^{3}$ (semiempirical medels) to $N^{6}$ (post Hartree-Fock models), where $N$ is the number of basis functions, quantum chemical methods are the solution of choice for gas phase systems comprising up to $10^{2}-10^{3}$ electrons. Some extensions of quantum chemistry techniques, taking account of periodicity conditions, have been suggested for solids such as crystals and polymers, and this is an extremely interesting development for solid-state chemists. It allows to calculate 